Because of the effect of time on allele burden in PV, we restricted clinical correlations to patients in whom JAK2V617F allele burden was performed within 5 years of diagnosis $(n=77$, median age at diagnosis 66 years, $43 \%$ women). Blood counts at presentation in these 77 patients included medians of $18.6 \mathrm{~g} / \mathrm{dl}$ for hemoglobin, $439 \times 10^{9} / \mathrm{l}$ for platelet count and $11.8 \times 10^{9} / \mathrm{I}$ for leukocyte count. At a median follow-up of 38 months, eight deaths, one leukemic transformation and two post-PV myelofibrosis were documented. Arterial and venous thrombosis occurred in $10(13 \%)$ and four $(5 \%)$ patients at diagnosis and in $15(19.5 \%)$ and four (5\%) patients during follow-up, respectively.

Linear regression analysis for continuous variables revealed that JAK2V617F allele burden correlated directly with leukocyte count $(P<0.0001)$ but not with hemoglobin level $(P=0.39)$, platelet count $(P=0.85)$, or age $(P=0.85)$. Similarly, allele burden was not affected by gender $(P=0.9)$. Allele burden (considered as a continuous variable) was significantly higher in the presence of pruritus $(P=0.003)$ or microvascular symptoms $(P=0.03)$ but not palpable splenomegaly $(P=0.89)$ or cytotoxic therapy during follow-up $(n=57$ versus 20 patients not receiving such therapy; $P=0.3$ ). Mutant allele burden was similar between the 14 patients who presented with major thrombosis (both arterial and venous) and the 63 patients who did not $(P=0.32)$; similarly, allele burden was not significantly different in those patients who developed major thrombosis during follow-up $(n=19)$ compared to those who did not $(n=58 ; P=0.95)$. The results did not change when arterial thrombosis $(P=0.38$ at diagnosis and 0.75 during follow-up) was analyzed separately from venous thrombosis $(P=0.51$ at diagnosis and 0.49 during follow-up). The above-mentioned results regarding clinical correlations of bone marrow JAK2V617F allele burden remained the same, for the most part, when allele burden was treated as a categorical variable with either two groups above and below the median value or three groups with allele burdens upto $25 \%(n=55)$, between 25 and $50 \%(n=10)$ and above $50 \%(n=12)$, respectively. The only different outcome was the loss of significant correlation involving microvascular symptoms and pruritus when allele burden was categorized into three groups. Finally, the number of events in terms of death, leukemic transformation, or development of post-PV myelofibrosis was too small to allow valid statistical comparisons of survival or disease transformation.

Taken together, the findings from the current study are underwhelming in terms of clinical relevance of bone marrow JAK2V617F allele burden in PV. The strongest and most consistent correlation was leukocytosis, an observation that warrants the inclusion of leukocyte count as a covariate in any statistical analysis of prognostic relevance that includes JAK2V617F allele burden. This result is pertinent to the recent emphasis on the prognostic relevance of leukocytosis in both PV and $\mathrm{ET}^{7}$ Our findings also underline the potential confounding effect of sample accrual time on result interpretation. Finally, we underscore the fact that the current study utilized DNA from archived bone marrow and should not be compared with other studies that have used either $D_{N A}^{2}$ or mRNA (Vannucchi AM et al. Blood 2006; 108: Abstract no. 5) from purified granulocytes.

A Tefferi $^{1}$, J Strand ${ }^{1}$, TL Lasho $^{1}$, RA Knudson ${ }^{2}$, CM Finke ${ }^{1}$, $N$ Gangat $^{1}$, A Pardanani $^{1}$, CA Hanson ${ }^{3}$ and RP Ketterling ${ }^{2}$ ${ }^{1}$ Division of Hematology, Mayo Clinic, Rochester, MN, USA;

${ }^{2}$ Laboratorie of Cytogenetics, Mayo Clinic, Rochester, MN, USA and

${ }^{3}$ Laboratorie of Hematopathology, Mayo Clinic, Rochester, $M N$, USA

E-mail: tefferi.ayalew@mayo.edu

\section{References}

1 Scott LM, Tong W, Levine RL, Scott MA, Beer PA, Stratton MR et al. JAK2 exon 12 mutations in polycythemia vera and idiopathic erythrocytosis. N Engl J Med 2007; 356: 459-468.

2 Tefferi A, Lasho TL, Schwager SM, Strand JS, Elliott M, Mesa R et al. The clinical phenotype of wild-type, heterozygous, and homozygous JAK2V617F in polycythemia vera. Cancer 2006; 106: 631-635.

3 Vannucchi AM, Pancrazzi A, Bogani C, Antonioli E, Guglielmelli P. A quantitative assay for JAK2(V617F) mutation in myeloproliferative disorders by ARMS-PCR and capillary electrophoresis. Leukemia 2006; 20: 1055-1060.

4 Levine RL, Belisle C, Wadleigh M, Zahrieh D, Lee S, Chagnon P et al. X-inactivation-based clonality analysis and quantitative JAK2V617F assessment reveal a strong association between clonality and JAK2V617F in PV but not ET/MMM, and identifies a subset of JAK2V617F-negative ET and MMM patients with clonal hematopoiesis. Blood 2006; 107: 4139-4141.

5 Gale RE, Allen AJ, Nash MJ, Linch DC. Long-term serial analysis of $\mathrm{X}$-chromosome inactivation patterns and JAK2 V617F mutant levels in patients with essential thrombocythemia show that minor mutantpositive clones can remain stable for many years. Blood 2007; 109: $1241-1243$

6 Kittur J, Knudson RA, Lasho TL, Finke CM, Gangat N, Wolanskyj AP et al. Clinical Correlates of JAK2V617F Allele Burden in Essential Thrombocythemia. Cancer 2007; in press.

7 Gangat N, Wolanskyj AP, McClure RF, Li CY, Schwager S, Wu W et al. Risk stratification for survival and leukemic transformation in essential thrombocythemia: a single institutional study of 605 patients. Leukemia 2007; 21: 270-276.

\title{
History of infections and vaccinations and risk of lymphoid neoplasms: does influenza immunization reduce the risk?
}

Leukemia (2007) 21, 2075-2079; doi:10.1038/sj.leu.2404738; published online 17 May 2007

Lymphoid neoplasms (LN) are malignancies of lymphocytes, the primary effectors of the immune system. LN are a heterogeneous group including non-Hodgkin's lymphoma (NHL), Hodgkin's lymphoma (HL), multiple myeloma (MM) and chronic lymphocytic leukaemia (CLL) and comprises numerous histological subtypes integrated in the third edition of the WHO International Classification in Oncology since 2001. Altogether, for the year 2000, the annual number of incident cases of LN in France was estimated to be over 17000 and the number of deaths to almost 9000. The incidence of NHL increased more than $3 \%$ 
yearly during the last two decades (1978-2000). Changes in diagnosis and registration practices over time and emergence of the human immunodeficiency virus (HIV) contributed to the rise of NHL but are insufficient to explain entirely observed trends. The aetiology of $\mathrm{LN}$ is largely unknown, especially for NHL, although various risk factors explain a small proportion of the incident cases worldwide. The present study has been conducted to investigate the potential relationship between $\mathrm{LN}$ and infections, environmental and occupational exposures and family history. This paper focuses on questions related to infections and immunizations, prevalence of which is likely to have substantially changed over time.

Several studies have shown that selected aspects of a history of infection and vaccination may be related to the risk of $\mathrm{LN}$. $^{1-5}$ The results are heterogeneous, varying with the type of infectious disease considered and with the histologic analysis of NHL as a whole or by subtype. A history of infectious mononucleosis was shown to be positively associated with $\mathrm{NHL},{ }^{1,2}$ but negatively associated with follicular lymphoma (FL) and diffuse large B-cell lymphoma (DLCL), in the only histologic subtype analysis, which was conducted by Holly et al. ${ }^{4} \mathrm{~A}$ medical history of herpes (labial) or hepatitis has been reported to be negatively related to NHL considered by subtype (DLCL and $\mathrm{FL}$ ), but analyses conducted on all NHL generated nonsignificant results or a positive association. ${ }^{2,3}$ Few results for bacterial infections are available: scarlet fever, pneumonia, pyelonephritis, tuberculosis and chronic infection have been reported to constitute an NHL risk. ${ }^{1-3,5}$ However, those findings were based on single-studies, or non-significant associations, or were not confirmed by histologic subtype analysis. ${ }^{4}$ Foreign travel does not seem to be associated with $\mathrm{NHL} .{ }^{4}$ The results for a history of malaria are contradictory. ${ }^{3,5}$

Immunization history also modulates immune regulation. In two case-control studies, ${ }^{3,4}$ there was a consistent pattern of decreased NHL risk, by disease subtype, associated with vaccinal history, except for BCG vaccination. Neither of the published studies included French data.

Between 2000 and 2004, we conducted a multicentre hospital-based case-control study (Bordeaux, Brest, Caen, Lille,
Nantes and Toulouse) including 824 cases and 752 controls. Eligible case were 18-75 years old, hospitalized for a recent diagnosis of non-Hodgkin's lymphoma $(n=399), \mathrm{HL}(n=149)$, MM ( $n=108)$ or lymphoproliferative syndromes (LPS; $n=168)$, including CLL and hairy cell leukaemia. Patients infected by HIV or treated by immunosuppressive drug were not eligible. Controls were hospitalized for any cause except a cancer, an accident or a disease directly related to occupation, tobacco or alcohol consumption and individually matched with cases by centre, gender, age ( \pm 3 years) and residential area. Face-to-face interviews were performed by trained interviewers using a standardized questionnaire on different topics including sociodemographic characteristics and personal medical history (occurrence of viral, bacterial diseases, common infection in childhood, chronic infection, tropical or parasitic diseases occurred during foreign travels). All the analyses were conducted using STATA software (version 8.2). The paired matching was broken for most analyses to gain power, using more controls per cases. Odds ratios (OR) were estimated using unconditional logistic regression and adjusted for matching variables. Polytomous logistic models were used to estimate OR jointly for the main NHL subgroups.

Age and sex were similarly distributed between cases and controls, all cases taken together. All strata comprised at least one control by case. Cases and controls were comparable according to their socio-professional categories and educational level, except for HL cases who were less often factory workers than controls.

Table 1 reports the association between history of infections based on patient's interview and large classes of LN. Infectious mononucleosis was significantly associated with $\mathrm{HL}(\mathrm{OR}=2.6$ [1.3-5.3]), and the association was stronger for men $(\mathrm{OR}=4.7$ [1.7-13.1]) than women $(\mathrm{OR}=1.4[0.5-3.9])$. There was no particular relationship with the age of mononucleosis occurrence. Only two cases and two controls had declared having had a mononucleosis after the age of 30 years. None of the LN was related with a history of herpes (cold sore or genital herpes) or shingles. ORs associated with a reported history of hepatitis of any type were slightly lower than the unit. No relationship was

Table 1 Relationship between history of infectious diseases and lymphoid neoplasms

\begin{tabular}{|c|c|c|c|c|c|c|c|c|c|c|c|c|c|c|c|c|c|c|c|}
\hline & \multicolumn{5}{|c|}{ LPS } & \multicolumn{5}{|c|}{ NHL } & \multicolumn{5}{|c|}{$H L$} & \multicolumn{4}{|c|}{$M M$} \\
\hline & $\mathrm{Ca}$ & Co & OR & $95 \% \mathrm{Cl}$ & $\mathrm{P}$ & $\mathrm{Ca}$ & Co & OR & $95 \% \mathrm{Cl}$ & $\mathrm{P}$ & $\mathrm{Ca}$ & Co & $O R$ & $95 \% \mathrm{Cl}$ & $P$ & $\mathrm{Ca}$ & Co & OR & $95 \% \mathrm{Cl}$ \\
\hline Mononucleosis & 1 & 5 & 0.6 & {$[0.1-5.3]$} & & 12 & 21 & 1.0 & {$[0.5-2.2]$} & & 21 & 20 & 2.6 & {$[1.3-5.3]$} & ** & 1 & 9 & 0.5 & {$[0.1-4.0]$} \\
\hline Cold sore & 64 & 159 & 0.9 & [0.6-1.4] & & 133 & 231 & 1.1 & {$[0.8-1.4]$} & & 46 & 131 & 1.0 & [0.6-1.5] & & 36 & 148 & 1.2 & [0.7-1.9] \\
\hline Genital herpes & 2 & 11 & 0.5 & {$[0.1-2.5]$} & & 10 & 15 & 1.1 & {$[0.5-2.4]$} & & 2 & 10 & 0.9 & {$[0.2-4.7]$} & & 4 & 12 & 1.7 & {$[0.5-5.5]$} \\
\hline $\begin{array}{l}\text { Cold sore or } \\
\text { genital herpes }\end{array}$ & 65 & 169 & 0.9 & [0.6-1.3] & & 139 & 242 & 1.1 & [0.8-1.4] & & 48 & 138 & 1.0 & [0.6-1.5] & & 39 & 157 & 1.2 & [0.8-2.0] \\
\hline Hépatitis & 17 & 64 & 0.7 & [0.4-1.2] & & 38 & 87 & 0.7 & {$[0.5-1.1]$} & & 11 & 51 & 0.6 & [0.3-1.3] & & 11 & 57 & 0.8 & [0.4-1.7] \\
\hline Mumps & 69 & 184 & 1.1 & [0.7-1.6] & & 181 & 303 & 1.1 & {$[0.8-1.4]$} & & 61 & 208 & 0.7 & {$[0.4-1.1]$} & & 38 & 205 & 0.8 & [0.5-1.3] \\
\hline Measles & 83 & 206 & 1.1 & {$[0.7-1.7]$} & & 193 & 322 & 1.2 & [0.9-1.6] & & 71 & 186 & 1.4 & [0.9-2.2] & & 46 & 226 & 0.8 & {$[0.5-1.2]$} \\
\hline Scarlet fever & 19 & 55 & 0.9 & {$[0.5-1.7]$} & & 37 & 81 & 0.8 & {$[0.5-1.2]$} & & 8 & 54 & 0.4 & {$[0.2-0.8]$} & * & 7 & 53 & 0.6 & {$[0.3-1.4]$} \\
\hline Tuberculosis & 11 & 32 & 1.0 & [0.5-2.0] & & 22 & 34 & 1.1 & {$[0.6-2.0]$} & & 4 & 6 & 4.5 & {$[1.2-17]$} & * & 4 & 26 & 0.7 & [0.2-2.0] \\
\hline $\begin{array}{l}\text { Sexually } \\
\text { transmitted diseases }\end{array}$ & 0 & 8 & - & - & & 11 & 16 & 1.2 & [0.5-2.6] & & 6 & 12 & 1.6 & [0.5-4.6] & & 1 & 12 & 0.4 & [0.1-3.6] \\
\hline \multicolumn{20}{|l|}{ Chronic infections } \\
\hline Ears nose throat & 53 & 117 & 1.3 & {$[0.9-1.9]$} & & 102 & 169 & 1.1 & {$[0.8-1.4]$} & & 30 & 105 & 0.7 & {$[0.4$} & & 31 & 113 & 1.4 & [0.8-2.2] \\
\hline Wound & 10 & 25 & 0.9 & [0.4-2.0] & & 12 & 31 & 0.7 & {$[0.3-1.4]$} & & 3 & 13 & 0.7 & {$[0.2-2.6]$} & & 6 & 23 & 1.3 & {$[0.5-3.4]$} \\
\hline Dental & 26 & 63 & 1.3 & [0.8-2.3] & & 54 & 85 & 1.1 & {$[0.7-1.6]$} & & 17 & 49 & 1.3 & {$[0.7-2.4]$} & & 15 & 70 & 1.0 & {$[0.5-1.8]$} \\
\hline
\end{tabular}

Abbreviations: HL, Hodgkin's lymphoma; LPS, lymphoproliferative syndrome; MM, multiple Myeloma; NHL, non-Hodgkin's lymphoma.

Odds ratios (OR) and $95 \%$ confidence intervals $(95 \% \mathrm{Cl}$ ) are estimated by unconditional logistic regression including the stratification variables age, gender and centre.

${ }^{\star} P<0.05 ;{ }^{\star \star} P<0.01$. 


\begin{tabular}{|c|c|c|c|c|c|c|c|c|c|c|c|c|c|c|c|c|c|c|c|c|}
\hline & \multicolumn{5}{|c|}{ LPS } & \multicolumn{5}{|c|}{$N H L$} & \multicolumn{5}{|c|}{$H L$} & \multicolumn{5}{|c|}{$M M$} \\
\hline & $\mathrm{Ca}$ & Co & $O R$ & $95 \% \mathrm{Cl}$ & $P$ & $\mathrm{Ca}$ & Co & $O R$ & $95 \% \mathrm{Cl}$ & $P$ & $\mathrm{Ca}$ & Co & $O R$ & $95 \% \mathrm{Cl}$ & $P$ & $\mathrm{Ca}$ & Co & $O R$ & $95 \% \mathrm{Cl}$ & P \\
\hline$B C G$ & 128 & 369 & 0.7 & [0.4-1.3] & & 321 & 576 & 1.0 & [0.6-1.5] & & 133 & 365 & 1.4 & [0.5-3.8] & & 82 & 377 & 1.6 & {$[0.7-3.4]$} & \\
\hline Hepatitis & 29 & 89 & 0.9 & {$[0.5-1.4]$} & & 86 & 176 & 0.8 & [0.6-1.2] & & 65 & 133 & 1.0 & [0.6-1.5] & & 20 & 95 & 0.9 & [0.5-1.6] & \\
\hline Cholera & 14 & 50 & 0.9 & {$[0.5-1.7]$} & & 30 & 71 & 0.7 & [0.4-1.1] & & 10 & 39 & 0.8 & [0.4-1.8] & & 11 & 48 & 1.1 & [0.5-2.3] & \\
\hline Yellow fever & 35 & 77 & 1.6 & [1.0-2.5] & & 69 & 110 & 1.1 & [0.8-1.5] & & 23 & 64 & 1.3 & {$[0.7-2.2]$} & & 23 & 82 & 1.4 & {$[0.8-2.4]$} & \\
\hline Typhoid & 21 & 48 & 1.6 & [0.9-3.0] & & 40 & 68 & 1.0 & [0.6-1.5] & & 13 & 32 & 1.5 & [0.7-3.2] & & 17 & 53 & 1.7 & [0.9-3.1] & \\
\hline Influenza & 69 & 219 & 0.7 & [0.4-1.0] & * & 130 & 258 & 0.8 & {$[0.6-1.1]$} & & 30 & 104 & 1.2 & {$[0.7-2.1]$} & & 46 & 204 & 0.9 & [0.6-1.5] & \\
\hline Never & 99 & 243 & 1.0 & Ref. & & 264 & 441 & 1.0 & Ref. & & 118 & 312 & 1.0 & Ref. & & 62 & 274 & 1.0 & Ref. & \\
\hline $1-5$ & 44 & 129 & 0.7 & {$[0.5-1.1]$} & & 86 & 151 & 0.9 & {$[0.7-1.3]$} & & 23 & 65 & 1.3 & {$[0.8-2.4]$} & & 35 & 120 & 1.2 & {$[0.7-2.0]$} & \\
\hline $\begin{array}{l}>5 \\
P \text { for trend }\end{array}$ & 23 & 86 & 0.6 & $\begin{array}{l}{[0.3-1.0]} \\
0.06\end{array}$ & * & 43 & 101 & 0.6 & $\begin{array}{l}{[0.4-1.0]} \\
0.05\end{array}$ & * & 6 & 34 & 0.9 & [0.3-2.5] & & 10 & 79 & 0.5 & $\begin{array}{l}{[0.2-1.0]} \\
0.04\end{array}$ & * \\
\hline
\end{tabular}

Abbreviations: HL, Hodgkin's lymphoma; LPS, Iymphoproliferative syndrome; MM, multiple myeloma; NHL, non-Hodgkin's lymphoma.

Odds ratios $(\mathrm{OR})$ and $95 \%$ confidence intervals $(95 \% \mathrm{Cl})$ are estimated by unconditional logistic regression including the stratification variables age, gender and centre.

${ }^{*} P<0.05$.

observed between LN and any childhood diseases (measles, mumps, chicken pox and scarlet fever) but the high number of missing values (15-22\%, according to the disease considered, similar for cases and controls) precluded detailed analysis. An association between $\mathrm{HL}$ and history of tuberculosis was observed with an OR of 4.5 [1.2-17.4] based on only four cases and six controls. There was no association with any chronic infections for any groups of LN. No association was observed, either, with reported history of common infections in childhood and young adult $(<10,<20,<30$ and $\geqslant 30$ years old) or with any groups of LN.

We observed an inverse relationship between vaccination against influenza and LPS, NHL and MM (Table 2), significant for LPS $(O R=0.7$ [0.4-1.0]). The ORs decreased with increasing the number of injections. Similar trends, were observed for CLL (0.01) and the two main subtypes of NHL, DLCL and FL although not significant. Analysis of usual vaccines such as polio, tetanus and smallpox was impossible due to the high number of missing values. No association was observed for other immunization except a positive association between yellow fever and LPS (border line of significance).

Associations were observed between LPS and foreign travels to Africa $(\mathrm{OR}=2.3[1.5-3.4])$ and Asia $(\mathrm{OR}=1.6[1.0-2.5])$, $\mathrm{MM}$ and travels to Africa $(\mathrm{OR}=1.6[1.0-2.5])$ and $\mathrm{HL}$ and travels to Asia, $(\mathrm{OR}=2.0$ [1.2-3.5]). We did not find any relationship with duration of the travel. HL patients were more likely to have got a disease when travelling abroad than controls $(\mathrm{OR}=1.8[1.0-3.2])$. None of the LN types was associated with a history of malaria. A history of gastroenteritis contracted during a journey in Africa or Asia was reported more often by $\mathrm{HL}, \mathrm{NHL}$ and MM cases and the association between gastroenteritis contracted in Asia and NHL was statistically significant $(\mathrm{OR}=2.6[1.3-5.1])$. The association was not restricted to a particular NHL subgroup.

Adjustment for socioprofessional categories and educational level did not change any of the results. Neither did the results change when conditional nor polytomous regression models were used to test the robustness of the observations. Results also remained stable when we repeated the analyses excluding any centres or any categories of causes of admission of controls to appreciate their weight in the estimations. Furthermore, the relationships between influenza immunization and LPS, NHL and $M M$ were reinforced when the analyses were performed excluding immunizations received before the last 5 years before diagnosis or interview, with ORs of 0.6 [0.4-1.0], 0.7 [0.5-0.9] and $0.5[0.3-0.9]$, respectively. Conversely, no association was observed when considering only the immunization received during the last 5 years. Finally, to avoid residual confounding by age, we repeated the analyses using narrower categories of age (3 years) to adjust for, and this did not change the results.

Various points of this study can be discussed: all were incident cases, which were systematically contacted by the interviewers and only 48 (5.5\%) refused the interview. Except for LPS whose good prognosis allowed that the interview could take place 1 year after the diagnosis, they were contacted early after the diagnosis to avoid selection on survival. Ninety per cent of the cases were diagnosed within 6 months. There is no obvious reason for patient's selection being related to vaccine status or past infectious disease. Besides, such selection would unlikely affect similarly all centres, and we checked by sensitivity analysis that the results were not modified by oneby-one exclusion of each centre. Cases were classified after pathologist's ascertainment according to the WHO classification. Subgroup analysis was performed whenever allowed by number of cases.

Controls have been mostly selected in orthopaedic and rheumatologic departments. Except for HL cases, who were as expected less often factory workers and had got a higher degree than controls, cases and controls did not differ according to their socioprofessional categories and educational level. To prevent a selection of patients with a lower socioprofessional category than cases and an over-representation of factors of interest in controls, patients admitted for a disease directly related to tobacco smoking, alcohol or occupation were not eligible as controls, although they may have had a history of such disease in the past. None of the causes of admission of the controls is known to be a particular indication for vaccination, or to be related to a particular prevalence of past infectious diseases. In addition, the results were not affected by one-by-one exclusion of each category of cause of admission of the controls. Prevalence of influenza immunization observed in controls was close to that of the French general population, which has been evaluated in 2000 by the national group of influenza Surveillance (http://www.grog.org/documents/2001). The vaccination coverage was estimated of $22 \%$ for the general population, close to the prevalence of $25 \%$ observed in our control group. The prevalence of vaccination was identical (57\%) for subjects aged 65-69 years, and a little lower for those 
aged $70-74$ years $(60 \%$ in the control group versus $66 \%$ in the general population). The results remained unchanged when the analysis was restricted to the highest age categories.

All our results rely on self-reported medical history and subsequently on patient recall. To minimize recall bias, we used a standardized questionnaire administered in same conditions to cases and controls by trained interviewers. Poor recall precluded investigation of usual infections and immunizations that had taken place during childhood and early adulthood. Recall seemed much better regarding less common diseases such as hepatitis or infectious mononucleosis, and more recent immunizations like that against flu, which data were rarely missing in cases as well as in controls. When we applied a 5-year lag excluding recent immunizations or infections that may have been related to the disease, the results were reinforced.

Influenza vaccination is not a known risk factor of cases or control diseases, making unlikely a voluntary understatement of the exposures by cases or an overstatement by controls. There is no obvious reason, either, for controls to have a better recall of their immunizations than cases. An underestimation of histories of infection for cases and controls is highly probable for many infections which can be forgotten or never known by the subjects themselves. This may be true for immunizations but less likely for repeated immunizations which were usually more recent and may be easier to remember. The relationship observed with the number of injections could be attributed to residual confounding by age, if controls were older than cases, the oldest subjects having received more injections than the youngest. Neither the analysis using strata of 3 years of age instead of 5, nor conditional analysis with individual matching for age \pm 3 years, changed the results. Socioprofessional categories or level of education were considered possible confounding factors, even though these variables were comparable between cases and controls, because they may influence the real prevalence of immunizations and infections as well as the recall and knowledge of personal medical history. These adjustments did not modify any of the results.

The positive association observed between $\mathrm{HL}$ and a history of mononucleosis was expected and of the same order of magnitude than that estimated by Hjalgrim et al. ${ }^{6}$ (standardized incidence ratio $=2.55$ [1.87-3.40]) from the follow-up of cohorts of mononucleosis patients.

The negative association between influenza immunization and LN has been previously reported by Holly et al. ${ }^{4}$ for several subtypes of non-Hodgkin's lymphoma. This study evidenced a significant inverse relationship between DLCL and influenza vaccination $(\mathrm{OR}=0.8[0.6-0.9])$. We report that ORs decreased with increasing number of injections of influenza vaccine for NHL and DLCL although non-significant for the latter. We also found a relationship for CLL and MM that had not been described previously, to our knowledge.

Other vaccines against various viral agents have been associated with a decreased risk of NHL: injectable polio vaccine in women, cholera vaccine in men and yellow fever vaccine in both men and women. We also observed OR lower than the unit associated with cholera vaccination for NHL, DLCL and CLL. The associations were not significant but it is noticeable that the prevalence of this vaccine was low in our population (about $10 \%$ in our controls versus $25 \%$ in the study by Holly et al. ${ }^{4}$ ). Conversely, to our knowledge, a positiveassociation between a vaccine against a virus agent and lymphoma (NHL and $\mathrm{HL}$ ) was only reported once, with oral polio vaccine in an Italian study, ${ }^{3}$ between oral polio vaccination and both NHL and HL. Influenza vaccine has been introduced in France mainly after the 1969 pandemic Hong-
Kong flu. It can be live, attenuated or inactivated (most often in France) and is recommended in elderly (more than 65 years), for patients with chronic diseases and health care professionals. In the present study, the association with influenza vaccination was stronger for at least five injections and when immunizations had taken place at least 5 years before diagnosis. These two results are linked and we cannot explain the magnitudes of these effects independently. Median of age at first vaccination was 55 years old (20-74 years).

Influenza vaccination by inactivated vaccine induces active immunity by stimulating systemic immune system. After injection, the influenza vaccine components are transported to draining lymph nodes in the armpit, either as free antigen, opsonized or carried by dendritic cells or other antigenpresenting cells. Depending on the formulation, whole or split inactivated influenza vaccine produces an immune response towards Th1 type of cytokines (IL-2 and IFN- $\gamma$ ) or Th2 type of cytokines (IL-4, IL-10) respectively. ${ }^{7}$ Repeated vaccinations could imbalance in the regulation and expression of Th1 and Th2 cytokines towards a Th1 thought to be protective against cancer. A shift in the balance of the Th1/Th2 response may play a role in aetiology of LNs as suggested by Holly et al. ${ }^{8}$ This hypothesis is also supported by recent findings that genetic variation in two key genes of Th2 pathway (IL-10, IL-4) could be associated with NHL.

The associations with gastroenteritis contracted when travelling in Africa or Asia have not been reported yet. Holly et al. ${ }^{4}$ did not find any association with foreign travel or contact with persons coming back from Africa. Becker et al. ${ }^{5}$ reported no association between malaria and NHL whereas Tavani et al. ${ }^{3}$ reported a positive relationship between malaria and NHL.

In conclusion, the present study showed no association between LNs and history of infectious disease except for the expected relationship between Hodgkin's lymphoma and mononucleosis. The inverse trend evidenced with influenza immunization, already described in a large previous study, deserves further investigation.

\section{Acknowledgements}

This work was supported by grants from the Association pour la Recherche contre le Cancer, the Fondation de France, and AFSSET and received a gift from Faberge employees. We are grateful to Sandra Leguyader-Peyrou, Marie-Astrid Caillet, Satya GarnierHaoussine, Virginie Duchenet, Véronique Chaigneau, Anne-Laure Demarty, Dominique Gillet and Magali Viaud who contributed to the interviews and to Martine Valdes, Christine Henry, Nathalie Jourdan-Da Silva and Dominique Ridondelli for technical assistance. We also thank the heads of department who helped us to include their patients as controls: Profs. Vital, Durandeau, Le Guillou at Bordeaux, Lefevre and Le Goff at Brest, Vielpeau and Marcelli at Caen, Migaux, Duquesnois, Mazeman at Lille, Passuti and Maugars at Nantes, Mansat and Fournier at Toulouse, and to Isabelle Soubeyran who helped the revision of the diagnoses with the staff of the Haematological malignancies registry of Gironde who helped for classification and coding. We are grateful to Andrew Mullarky for his skillful revision of this paper.

$$
\begin{aligned}
& \text { A Monnereau }{ }^{1,2,3}, \text { L Orsi }^{1,2}, \text { X Troussard }^{4}, \mathrm{C} \mathrm{Berthou}^{5} \\
& \text { P Fenaux }{ }^{6}, \text { G Marit }^{7^{\prime}}, \text { P Soubeyran }^{3}, \text { F Huguet }^{8^{\prime}}, \\
& \mathrm{N} \text { Milpied }^{9}, \mathrm{M} \text { Leporrier }^{4},{ }^{2} \text { D Hemon }{ }^{1,2} \text { and J Clavel }{ }^{1,2} \\
& { }^{1} \text { INSERM, U754, Villejuif, France; } \\
& { }^{2} \text { Université Paris-Sud, UMR-S754, IFR69, Villejuif, France; } \\
& { }^{3} \text { Institut Bergonié, Centre de Lutte Contre le Cancer, }
\end{aligned}
$$

Bordeaux, France; 
${ }^{4}$ Laboratoire d'hématologie, Hôpital Côte de Nacre, Caen, France;

${ }^{5}$ Département d'hématologie, Hôpital Morvan, Brest, France

${ }^{6}$ Département d'hématologie, Hôpital Avicenne, Bobigny,

France;

${ }^{7}$ Service des maladies du sang, Hôpital du Haut-Lévêque, Pessac, France;

${ }^{8}$ Département d'hématologie, Hôpital Purpan, Toulouse, France and

${ }^{9}$ Département d'hématologie, Hôtel Dieu, Nantes, France E-mail: monnereau@bergonie.org

\section{References}

1 Cartwright RA, McKinney PA, O'Brien C, Richards ID, Roberts B Lauder I et al. Non-Hodgkin's lymphoma: case control epidemiological study in Yorkshire. Leuk Res 1988; 12: 81-88.

2 Vineis P, Crosignani P, Sacerdote C, Fontana A, Masala G, Miligi L et al. Haematopoietic cancer and medical history: a multicentre case-control study. J Epidemiol Community Health 2000; 54: 431-436.

3 Tavani A, La Vecchia C, Franceschi S, Serraino D, Carbone A. Medical history and risk of Hodgkin's and non-Hodgkin's lymphomas. Eur / Cancer Prev 2000; 9: 59-64.

4 Holly EA, Bracci PM. Population-based study of non-Hodgkin's lymphoma, histology, and medical history among human immunodeficiency virus-negative participants in San Francisco. Am J Epidemiol 2003; 158: 316-327.

5 Becker N, Deeg E, Rudiger T, Nieters A. Medical history and risk for lymphoma: results of a population-based case-control study in Germany. Eur J Cancer 2005; 41: 133-142.

6 Hjalgrim H, Askling J, Sorensen P, Madsen M, Rosdahl N, Storm HH et al. Risk of Hodgkin's disease and other cancers after infectious mononucleosis. I Natl Cancer Inst 2000; 92: 1522-1528.

7 Szyszko E, Brokstad K, Cox RJ, Hovden AO, Madhun A, Haaheim LR. Impact of influenza vaccine formulation with a detailed analysis of the cytokine response. Scand J Immunol 2006; 64: 467-475.

8 Lan Q, Zheng T, Rothman N, Zhang Y, Wang SS, Shen M et al. Cytokine polymorphisms in the Th1/Th2 pathway and susceptibility to non-Hodgkin's lymphoma. Blood 2006; 107: 4101-4108.

\section{Interstitial del(14)(q) involving IGH: a novel recurrent aberration in B-NHL}

Leukemia (2007) 21, 2079-2083; doi:10.1038/sj.leu.2404739; published online 24 May 2007

Deletions of chromosome 14 recurrently observed in B-cell malignancies are particularly frequent in chronic lymphocytic leukemia (CLL), multiple myeloma $(\mathrm{MM})$, diffuse large B-cell lymphoma (DLBCL) and B-cell acute lymphoblastic leukemia. ${ }^{1}$ To characterize these aberrations, we initially mapped the deletions in 23 leukemia/lymphoma cases using a tiling path chromosome 14-array $\mathrm{CGH}(\mathrm{aCGH})$ platform (resolution of approximately $42 \mathrm{~kb}$ ) comprising 838 bacterial artificial chromosome/P1 artificial chromosome (BAC/PAC) clones from the Chori 32K set (www.ensembl.org). $\operatorname{Del}(14)(q)$ have been successfully mapped in all 23 cases (Figure 1a) and subdivided into two main categories: (1) involving or (2) not involving 14q32.33. This latter category grouped eight cases with interstitial deletions ranging in size from 17 to $70 \mathrm{Mb}$ and distributed along chromosome $14(\mathrm{q} 13 \rightarrow$ ter) and one case with three dispersed deletions of $1.5,7.5$ and $2.5 \mathrm{Mb}$. The first category of del $(14)(q)$ comprised 14 cases showing the common distal breakpoint mapped in the 105.26-105.41 Mb region harboring the $I G H$ genes cluster. The size of the deleted region varied in six cases; their proximal breakpoints were bordered by RP11-164H3/95.19Mb, CTD-2053J06/83.56 Mb, RP11340F04/74.66 Mb， RP11-350H11/67.94 Mb，RP11-769O09/ $66 \mathrm{Mb}$ and RP11-520H13/65 Mb. Particularly intriguing was the finding of exactly the same $\operatorname{del}(14)(q 24.1 q 32.33)$ covering the region of approximately $36 \mathrm{Mb}$ in eight cases (Figure $1 \mathrm{~b}$ ). The proximal border of this deletion was consistently flanked by RP11-35D12 $(68.24 \mathrm{Mb})(\times 2)$ and RP11$720119(68.45 \mathrm{Mb})(\times 1)$, and the distal breakpoint was bordered by RP11-448N05 $(105.26 \mathrm{Mb})(\times 1)$ and RP11-284A08 $(105.43 \mathrm{Mb})(\times 2)$. Given that these terminal BACs are assigned to $I G C H$ and $I G V H$, respectively, aCGH results were additionally validated by fluorescence in situ hybridization (FISH) with the LSI-IGH break-apart probe. As expected, all eight analyzed cases (as well as the remaining six cases with nonrecurrent $I G H$ - involving deletions) showed a one-fusion one-green signal pattern due to loss of the $3^{\prime} I G H$ flanking sequences (red signal). The proximal breakpoint of the $\operatorname{del}(14)(\mathrm{q} 24.1 \mathrm{q} 32.33)$ validated with RP11-35D12 and RP11-720l19 showed lost or diminished signal from the latter clone (Figure $1 \mathrm{~b}$ ) and thereby pointed to a breakpoint in the region covered by the overlapping extremities of these BAC clones. The only gene located in this region was ZFP36L1. For FISH detection of the $\operatorname{del}(14)(\mathrm{q} 24.1 \mathrm{q} 32.33)$ in other B-cell non-Hodgkin's lymphoma (B-NHL), a dual-color break-apart assay for the 14q24.1 breakpoint (RP11-35D12-SO/ RP11-720I19-SG) and LSI-IGH were applied. The screening of 58 B-NHL cases with cytogenetic and/or FISH evidence of $\operatorname{del}(14)(q)$ led to identification of 13 additional cases with $\operatorname{del}(14)(q 24.1 q 32.33)$, including one with the deletion masked by $\mathrm{t}(14 ; 22)(\mathrm{q} 32 ; \mathrm{q} 11) / / G H-I G L$ (case 7$)$. Additional FISH screening of 60 random CLL cases, seven MM cell lines, including four (RPMI-8226, L-363, OPM-1 and LP-1) with a previously described del(14), ${ }^{2}$ and 20 various B-NHL cases with structural aberrations of 14q21-q24, failed to identify ZFP36L1 and/or $I G H$ rearrangements, indicating that these deletions are rare molecular events.

The relevant clinical data of 21 cases with the del(14)(q24.1q32.33) (index cases) are summarized in Table 1. There were 14 male and 7 female patients $(2.0 \mathrm{M} / \mathrm{F}$ ratio) ranging in age from 52 to 90 years (mean 71.6). These cases represented a spectrum of B-cell malignancies including typical CLL $(n=8)$, atypical CLL showing either morphological or immunophenotypic discordances (for example, expressing CD22 $(n=4)$ and/or FMC7 $(n=2)$, and/or lacking expression of CD23 $(n=2)$ or CD5 $(n=1))(n=7)$, low-grade B-NHL (marginal zone lymphoma (MZL), Waldenstrom macroglobulinemia (WM), not otherwise specified NHL (NOS-NHL)) $(n=3)$ and $M M(n=3)$. Case 17 , diagnosed as MZL, transformed to a fatal DLBCL 2 years after diagnosis. Among CLL patients, five presented in Binet stage $A$, two in stage $B$ and five in stage $C$ (no available staging data in two cases). The majority of patients $(18 / 21)$ required therapy and when given (alkylators), they responded initially (data not shown). Nine patients are alive 